\title{
Demonstration of Astrocytes in Cultured Amniotic Fluid Cells of Three Cases with Neural-Tube Defect
}

\author{
Marion Cremer ${ }^{1}$, Melitta Schachner ${ }^{2}$, Thomas Cremer $^{1}$, Werner Schmidt ${ }^{3}$, and Theda Voigtländer ${ }^{1}$ \\ ${ }^{1}$ Institut für Anthropologie und Humangenetik, Universität Heidelberg, Im Neuenheimer Feld 328, D-6900 Heidelberg, \\ Federal Republic of Germany \\ ${ }^{2}$ Institut für Neurobiologie, Universität Heidelberg, Im Neuenheimer Feld 504, D-6900 Heidelberg, Federal Republic of Germany \\ ${ }^{3}$ Universitätsfrauenklinik, 6900 Heidelberg, Thibautstrasse, Federal Republic of Germany
}

Summary. We have investigated the origin of rapidly adhering (RA) cells in three cases of neural tube defects (two anencephali, one encephalocele). We were able to demonstrate the presence of glial fibrillary acidic (GFA) protein in variable percentages (4-80\%) of RA cells cultured for 4-6 days by use of indirect immunofluorescence with GFA antiserum. Cells cultured from amniotic fluids of normal pregnancies and fetal fibroblasts were completely GFA protein negative. GFA protein is well established as a highly specific marker for astrocytes. Demonstration of astrocytes may prove to be a criterion of high diagnostic value for neural tube defects. The percentage of astrocytes decreased with increasing culture time, while the percentage of fibronectin positive cells increased both in amniotic fluid cell cultures from neural tube defects and normal pregnancies.

\section{Introduction}

It has been shown that certain cells cultured from amniotic fluid in cases of neural-tube defects (NTD) differ from epitheloid cells obtained from normal amniotic fluids (Sutherland et al. 1975). These cells show a different morphology and attach more rapidly to the surface of culture-plates and have therefore been called rapidly adhering (RA) cells (Gosden and Brock 1977; Voigtländer et al. 1978). Sutherland et al. (1975) have assumed that many of the amniotic fluid cells found in NTD are macrophages, while Gosden and Brock (1977) have proposed on the basis of morphological criteria that RA cells might be of neural origin.

Offprint requests to: $\mathrm{M}$. Cremer (address see above)
RA cells however have also been detected in other fetal malformations, such as exomphalos (Gosden and Brock 1978). A precise characterization of the tissue specific origin of such cells is therefore highly desirable. In the present study we have applied indirect immunofluorescence to investigate the origin of amniotic fluid cells in three cases of NTD by use of antisera to glial fibrillary acidic (GFA) protein and to fibronectin. GFA protein has been demonstrated to be a subunit of intermediate-sized filaments in astrocytes and a highly specific marker for this cell type (Eng et al. 1971; Eng and Kosek 1974). The GFA protein antiserum used in this study has been shown to react exclusively with filaments of astrocytes but not of other cell types including fibroblasts and neurons (Bignami and Dahl 1974; Schachner et al. 1977). Since a considerable percentage of RA cells shows a fibroblastoid morphology, fibronectin antiserum was used to detect fibronectin as a cell surface antigen mainly expressed in fibroblasts (Schachner et al. 1978).

\section{Cases and Methods}

Cases 1 and 2

An anencephalus was found by ultrasonographic examination in the 18th week of pregnancy of a 21-year-old primigravida (case 1) and the 25 th week of pregnancy of a 24 -year-old primigravida (case 2). Amniocentesis revealed an alpha-fetoprotein (AFP) level of $465 \mathrm{ug} / \mathrm{ml}$ in case 1 , which is almost 100 standard deviations (SD) above the mean for the 18 th week of pregnancy and an AFP value of $38 \mathrm{ug} / \mathrm{ml}$ which is about $4.5 \mathrm{SD}$ above the mean for the appropriate week in case 2. Gel electrophoresis of acetylcholinesterase (AChE) showed the fast band of an $\mathrm{AChE}$ isoenzyme in both cases which is typical although not specific for NTD (Buamah et al. 1980; Haas-Andela et al. 1980). The pregnancies were terminated in both cases and the diagnosis was confirmed. 

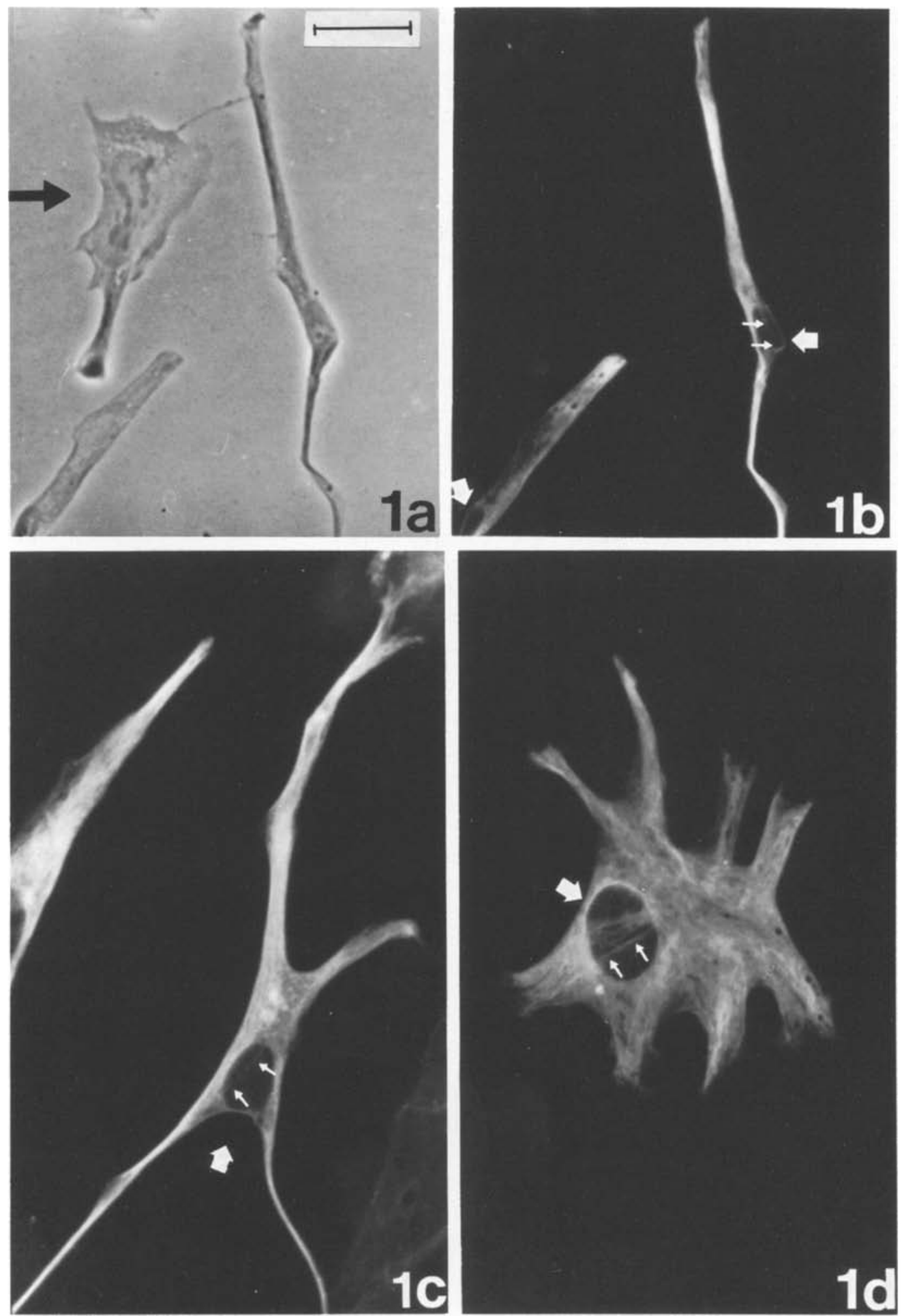

Fig. 1. GFA protein positive and negative cultured amniotic fluid cells from pregnancies with NTD. Phase contrast a) and immunofluorescent micrographs $(\mathbf{b}-\mathbf{f})$. Note the variable morphology of the cells. (Bar $10 \mathrm{um}$ ); $\mathbf{a}$ three cells in phase contrast; $\mathbf{b}$ the same cells with immunofluorescence, long bipolar type GFA protein positive cell, GFA protein negative cell (black arrow in a) not visible; c and d GFA protein cells with multiple processes; e and f GFA protein positive triangular cells; g GFA protein positive large multiangular cell. Note the following criteria for GFA protein positive cells: Nuclei spared from fluorescence (large white arrows), but occasional GFA protein positive filaments over the nuclei, fluorescent filamentous network in the cytoplasm 

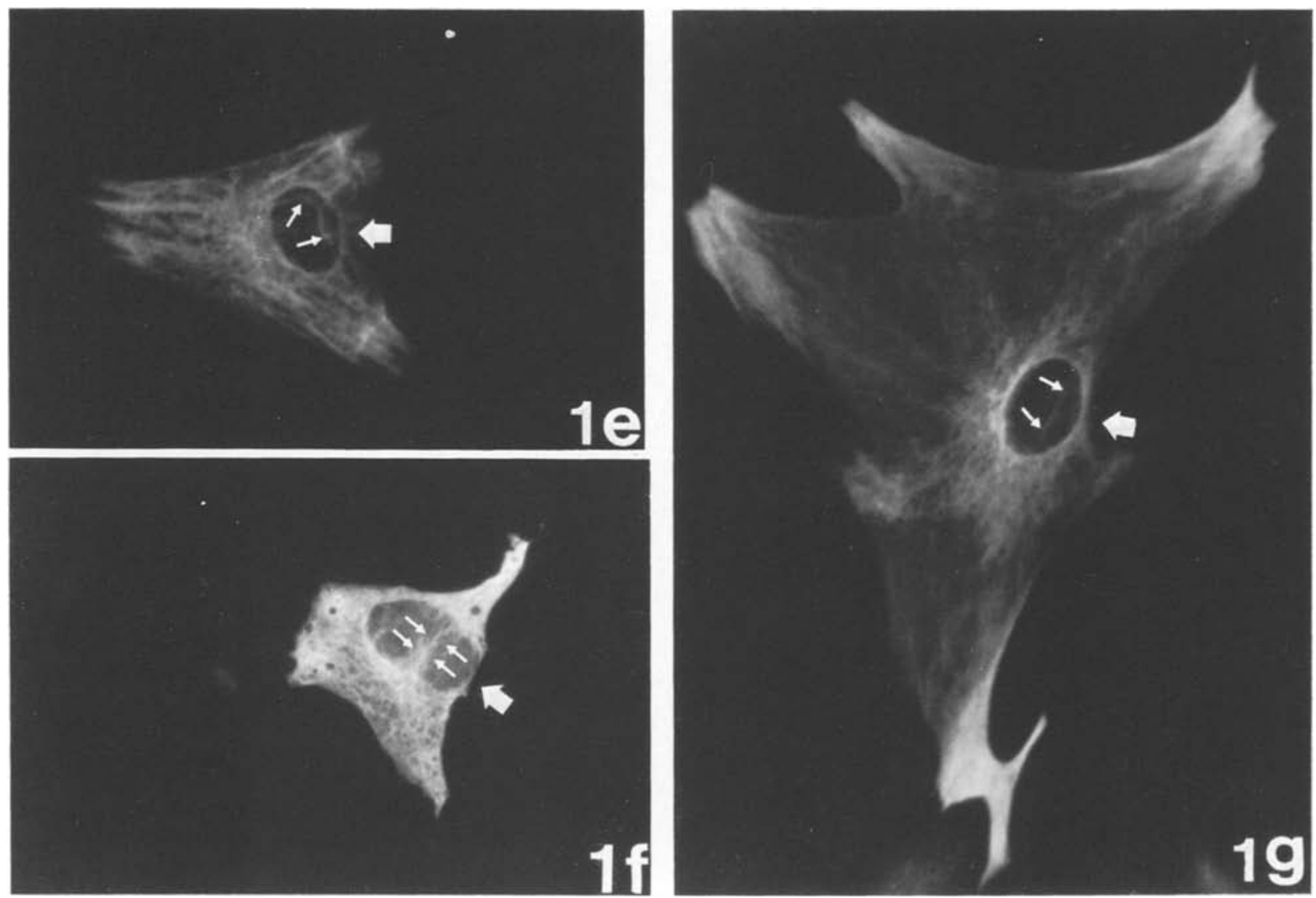

Case 3

This was the second pregnancy of a 22-year-old nullipara. The first pregnancy ended in a spontaneous abortion in the 11th week. In the 30 th week of this pregnancy ultrasonographic examination prompted the suspicion of some large tumor in the region of the head/neck, but an exact diagnosis was not possible. Amniocentesis resulted in an AFP level of $8.8 \mathrm{ug} / \mathrm{ml}$ which is above the 95th percentile for this week (Norgaard-Pedersen et al. 1976). The AChE pattern corresponded to that of cases 1 and 2. The diagnosis of an extensive NTD was strongly supported by the method described in this paper. The pregnancy was terminated after it became obvious that the fetus was not capable surviving and a large open encephalocele was found.

\section{Cultivation of Amniotic Fluid Cells}

Amniotic fluid from the three cases described above was centrifuged and the pellet of cells was suspended in HAM F 10 medium supplemented with $20 \%$ fetal calf serum (FCS), penicilline $(100 \mathrm{U} / \mathrm{ml})$ and streptomycine $(100 \mu \mathrm{g} / \mathrm{ml})$. Approximately $2 \times 10^{4}$ cells were seeded in each of a number of Leighton tubes with $10 \times 50 \mathrm{~mm}$ cover slips in a volume of $2.5 \mathrm{ml}$ medium at $\mathrm{pH}$ 7.3. Leighton tubes were sealed and incubated at $37^{\circ} \mathrm{C}$. Fresh medium was applied after $24 \mathrm{~h}$ in order to remove cells which had not adhered to the slides during this period of time. Culture medium of RA cells was changed every two days.

Amniotic fluid cells from the 17th week of pregnancy inconspicious for NTD were cultivated in the same way but changing of the medium was first done after five days of cultivation when epitheloid cells had attached to the glass.

\section{Fibroblast Cultures}

Small fascia biopsies were obtained from aborted fetuses of the 17 th-22nd week of gestation. One $\mathrm{mm}^{2}$ pieces were seeded between two cover slips in Leighton tubes and supplied with the same medium described above. After 4-6 weeks a layer of fibroblasts had formed. Cultures were used after the first or second passage.

\section{Determination of $A F P$}

AFP was quantitatively determined by immuno-electrophoresis according to the method of Laurell et al. (1966), using human $\alpha_{1-}$ fetoprotein antiserum and human $\alpha_{1}$-fetoprotein standard serum purchased from Behring (Marburg).

\section{Determination of AChE}

The amniotic fluid isoenzyme patterns of AChE were obtained using acrylamide gel electrophoresis as described by Smith et al. (1979).

\section{Indirect Immunofluorescence Technique}

Indirect immunofluorescence was carried out as described previously by Schnitzer and Schachner (1980). The cultured cells were gently washed in phosphate buffered saline (PBS), $\mathrm{pH} 7.3$, fixed with 4\% paraformaldehyde in PBS for $15 \mathrm{~min}$ and washed again twice in PBS, all at room temperature. The cultured cells were then incubated in absolute ethanol at $-20^{\circ} \mathrm{C}$ for $5 \mathrm{~min}$, washed again twice in PBS and treated with GFA protein antiserum ( $1: 300$ diluted) for $20 \mathrm{~min}$ at room temperature. Cells were 

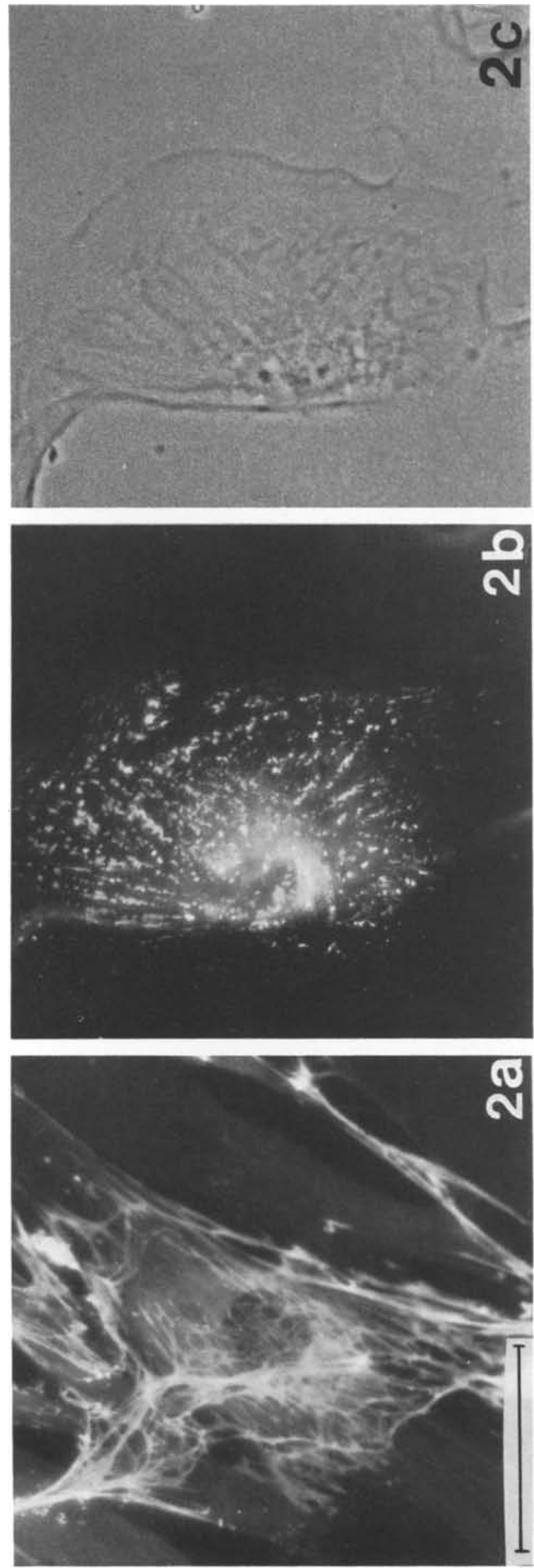

M. Cremer et al.: Astrocytes in Cultured Amniotic Fluid Cells

washed three times in PBS and incubated with goat anti-rabbit immunoglobulin coupled with fluorescein-isothiocyanate (GARIg-FITC 1:50, from Antibodies Inc.) for $20 \mathrm{~min}$, also at room temperature. Cultures were then washed again three times in PBS, mounted in glycerol-PBS $(1: 1, \mathrm{v} / \mathrm{v})$ and inspected in a Zeiss fluorescence microscope equipped with the appropriate filter. Indirect immunofluorescence with fibronectin antiserum was carried out in exactly the same way except that dilution was $1: 100$. Photographs were taken on a HP 4 film (22 DIN, Ilford).

\section{Results}

In three cases of NTD (two anencephali, one open encephalocele) we were able to demonstrate GFA protein positive cells in cultures established from the amniotic fluid after amniocentesis (Fig. 1). The positive cells showed intense cytoplasmic fibrillar fluorescence when stained by indirect immunofluorescence with antiserum to GFA protein while other cells lacked the GFA protein and did not stain (compare Fig. 1a,b). The morphology of GFA protein positive cells varied considerably as demonstrated by the examples shown in Fig. 1, ranging from large and small epitheloid cells to cells with long bipolar or multiple processes. GFA protein positive cells with similar morphology have been observed in primary cultures of early postnatal mouse cerebellum (Schnitzer and Schachner 1980). GFA protein positive cells could be clearly demonstrated by indirect immunofluorescence only after four days of cultivation. This effect cannot be due to a delayed attachment of astrocytes, since free cells were removed after $24 \mathrm{~h}$ of culture in vitro, but rather indicates a time of recovery needed to express sufficient quantities of GFA protein in the cells. The percentage of GFA protein positive cells found after four to six days ranged from $4 \%$ in case 1 (anencephalus) up to approximately $80 \%$ in case 3 (encephalocele). In case 2 the number of RA-cells was small and the cells had grown for 18 days before they were tested for GFA protein. In addition to a number of compact colonies composed of GFA negative but fibronectin positive fibroblast-like cells, we detected a sparse small colony of 23 GFA protein positive cells which showed a more epitheloid morphology. This indicates that astrocytes can be maintained and possibly even divide in the cultures for several weeks. Fibronectin positive cells amounted to a few percent only after four days of

Fig. 2. Immunofluorescent micrographs from fibronectin positive cells (Bar 10 um); a Typical fibronectin network in a human fetal fibroblast. The culture was established from fascia lata of a human fetus at the 22nd week of gestation; b Speckled fibronectin pattern in a cultured amniotic fluid cell with epitheloid morphology obtained from a normal pregnancy. Immunofluorescent staining was performed after a culture time of 18 days; $\mathbf{c}$ Cell from $2 b$ in phase contrast 
cultivation in vitro, but their number continously increased with time in culture at the expense of GFA protein positive cells.

The following diagnostic criteria of GFA protein positive cells might be useful as a guideline for those who are not familiar with immunofluorescence techniques. GFA protein positive cells exhibit a fine network of fluorescent filaments in the cytoplasm, while the region of nuclei is spared from reaction with the antiserum and thus remains completely dark (large white arrows in Fig. 1). The nuclear envelope is outlined by perinuclear fluorescent filaments. Occasional GFA protein positive bundles of filaments, which run in the cytoplasmic layer above or below the nuclei contrast clearly against the dark nuclear background (small arrows in Fig. 1). GFA protein negative cells may occasionally show some background fluorescence, especially when the excess of antibodies is not carefully removed. This unspecific fluorescence can easily be distinguished from the specific fluorescence by several criteria: it is diffuse, i.e. it does not exhibit the fine network of fluorescent cytoplasmic filaments and it does not spare the nuclei. Furthermore, it appears to be of brownish or greyish yellow colour rather than the brilliantly greenish fluorescence of GFA protein positive filaments. In addition background fluorescence intensity is very weak compared to GFA protein positive cells. Under appropriate technical conditions GFA protein negative cells do not stain at all, but even under less ideal conditions GFA protein positive cells can be unequivocally distinguished by these criteria from unspecifically fluorescing GFA protein negative cells.

Cultures established from amniotic fluids of normal pregnancies, and primary cultures of fetal fibroblasts were completely GFA protein negative. In contrast all the cells of the fetal fibroblast cultures showed a clear network of the cell membrane associated fibrillar fibronectin (Fig. 2 a). In normal cell cultures expression of fibronectin was only tested after several weeks of cultivation. A considerable proportion of these cells appeared to be fibronectin positive (Fig. 2b), but we have presently not quantitated these observations. Both, the speckled fibronectin pattern shown in Fig. $2 b$ and the fibronectin network shown in Fig. 2 a, was observed in cells of these cultures.

\section{Discussion}

Our results show that astrocytes were found in amniotic fluid cells from three fetuses with neural tube defects (NTD). This evidence is based on the demonstration of GFA protein positive filaments in cultured amniotic fluid cells by indirect immunofluorescence. GFA protein has previously been shown to be a highly specific marker protein of astrocytes in many species (Bignami and Dahl 1974; Eng and Kosek 1974; Eng et al. 1971; Schachner et al. 1977). It is important to stress that GFA protein is known to occur only in astrocytes and not any other neural or non-neural cell type investigated so far (Schachner et al. 1977). The proportion of cells that reacted with antiserum to fibronectin, a cell surface marker for fibroblasts and fibroblast-like cells (Schachner et al. 1978) was low in young cultures of NTD cases, but increased steadily with time in culture so that after several weeks in vitro hardly any GFA protein-positive cells were detectable. Amniotic cells obtained from normal pregnancies did not react with antiserum to GFA protein. Fibronectin-positive cells were also observed in cultures from normal amniotic fluids (Crouch et al. 1978), but it should be stressed that this cell marker has been found in certain epithelial cells (Chen et al. 1977) and does not allow any definite conclusion concerning the origin of the cells. The demonstration of astrocytes in amniotic fluids clearly indicates the presence of cells of neural origin in three cases of NTD. This effect is likely to depend on some direct contact of neural tissue with this fluid. We hope that this test might create another criterion of high diagnostic value for NTD. Since the test is performed on a single cell basis, detection of only a few astrocytes may strongly support the diagnosis of NTD. The validity of the test concerning the exclusion of NTD is limited by the fact that cell layers of non-astrocytic origin could prevent astrocytes from being sloughed off into amniotic fluid. Further investigations are necessary to quantitatively characterize the correlation of NTD and the demonstration of astrocytes in amniotic fluids. We would also like to stress the fact that between $20 \%$ and $96 \%$ of the amniotic cells tested after four days of cultivation did not show the GFA protein positive pattern in the three cases of NTD tested so far. While it seems possible that some astrocytes had not expressed GFA protein under the culture conditions used in these experiments, we assume that many of these cells were likely to be of non-astrocyte origin. In order to characterize these cells we are planning to test additional cell markers established for macrophages, neuronal cells and fibroblastic cells in future investigations. Interestingly, Sakar et al. (1980) have very recently reported on the presence of glial protein S-100 in rapidly adhering cells from a fetus with NTD.

Prenatal diagnosis of NTD is routinely performed by ultrasonography (Campbell and Thoms 1977) and by the investigation of biochemical markers in amniotic fluid, such as alpha-fetoprotein (AFP) (Wald and Cuckle 1979) and an acetylcholinesterase isoenzyme (Buamah et al. 1980; Smith et al. 1979). Each of these diagnostic procedures has presently its advantages and 
shortcomings. Small defects as well as defects situated in the low lumbar part of the spine may be missed by ultrasonography. Increased AFP values are not specific for NTD but can be found in a number of other pathological conditions as well, including congenital nephrosis or even such badly characterized conditions as "fetal distress" (Seppälä 1977). Even AChE does not seem to be exclusively related to NTD, as it has recently been demonstrated in several cases of exomphalos and intrauterine death (Buamah 1980). We have observed a case of a large exomphalos where the diagnosis was suggested by ultrasonography in the 16th week of pregnancy and has been confirmed after termination of the pregnancy. Amniotic fluid obtained by amniocentesis in this case showed elevated AFP levels $(220 \mu \mathrm{g} / \mathrm{ml})$ and was found to be $\mathrm{AChE}$ positive but we were not able to demonstrate any GFA protein positive cells in amniotic cell cultures (our unpublished data).

The basis of any responsible decision about terminating an abnormal pregnancy is the exact prenatal diagnosis. To make such a precise diagnosis the present observations may be useful in certain cases. Besides the possible significance of our results for prenatal diagnosis of NTD, it seems of general interest to exploit the possibilities of immunocytological methods to characterize cells from normal and pathological amniotic fluids.

Acknowledgements. The authors are indebted to Drs. L. F. Eng and R. O. Hynes for their generous gifts of antisera, to R. Zehnle for excellent technical assistance and to Prof. T.M.Schroeder and Prof. F. Vogel for their continous interest in this study. M.C. was supported by a Ausbildungsstipendium from the Deutsche Forschungsgemeinschaft and M.S. by a grant from Stiftung Volkswagenwerk.

\section{References}

Bignami D, Dahl D (1974) Astrocyte-specific protein and neuroglial differentiation. An immunofluorescent study with antibodies to the fibrillary acidic protein. J Comp Neurol 153: $27-38$

Buamah AK, Evans L, Ward AM (1980) Amniotic fluid acetylcholinesterase isoenzyme patterns in the diagnosis of neural tube defects. Clin Chim Acta 103: 147-151

Campbell S, Thoms A (1977) The use of ultrasound in the antenatal diagnosis of neural tube defects. Birth Defects: Orig Art Ser XII, No 3D:209-216

Chen LB, Maitland N, Gallimore PH, McDougall JK (1977) Detection of the large external transformation-sensitive protein on some epithelial cells. Exp Cell Res 106:39-46

Crouch E, Balian G, Holbrook K, Duskin D, Bornstein P (1978) Amniotic fluid fibronectin. J Cell Biol 78:701-715

Eng LF, Kosek JC (1974) Light and electron microscopic localization of the glial fibrillary acidic protein and S-100 protein by immunoenzymatic techniques. Trans Am Soc Neurochem 5:160

Eng LF, Vanderhaeghen JJ, Bignami A, Gerstl B (1971) An acidic protein isolated from fibrous astrocytes. Brain Res $28: 351-354$
Gosden CM, Brock DJH (1977) Morphology of rapidly adhering amniotic fluid cells as an aid to the diagnosis of neuraltube defects. Lancet I:919-922

Gosden CM, Brock DJH, Eason P (1977) The origin of the rapidly adhering cells found in amniotic fluids from foetuses with neural tube defects. Clin Genet 12:193-201

Gosden CM, Brock DJH (1978) Combined used of alphafetoprotein and amniotic fluid cell morphology in early prenatal diagnosis of fetal abnormalities. J Med Genet $15: 262-270$

Haas-Andela H, Altland K, Fuhrmann W, Bleyl H (1980) Screening und pränatale Diagnostik von Neuralrohrdefekten - Alpha-Fetoprotein-Spiegel und AcetylcholinesteraseMuster. Abstraktbuch der 2. Tagung der Arbeitsgemeinschaft klinische Genetik in Gießen

Laurell CB (1966) Quantitative estimation of proteins by electrophoresis in agarose gel containing antibodies. Anal Biochem 15:45

Nørgaard-Pedersen B, Jørgensen PJ, Trolle D (1976) Alphafetoprotein concentration in amniotic fluid during the last trimester in normal pregnancies and in pregnancies with severe fetal abnormalities. Acta Obstet Gynecol Scand 55: $59-62$

Sarkar S, Chang HC, Porreco RP, Jones OW (1980) Neural origin of cells in amniotic fluid. Am J Obstet Gynecol 136: 67-72

Schachner M, Hedley-White ET, Hsu DW, Schoonmaker G, Bignami A (1977) Ultrastructural localization of glial fibrillary acidic protein in mouse cerebellum by immunoperoxidase labeling. J Cell Biol 75:67-73

Schachner M, Schoonmaker G, Hynes RO (1978) Cellular and subcellular localization of LETS protein in the nervous system. Brain Res 158:149-158

Schnitzer J, Schachner M (1980a) Expression of Thy-1, H-2 and NS-4 cell surface antigens and tetanus toxin receptors in early postnatal and adult mouse cerebellum. Submitted for publication

Schnitzer J, Schachner M (1980b) Characterization of isolated mouse cerebellar cell population in vitro. Submitted for publication

Seppälä M (1977) Immunologic detection of alpha-fetoprotein as a marker of fetal pathology. Clin Obstet Gynecol 20: 737-757

Smith AD, Wald NJ, Cuckle HS, Stirrat GM, Bobrow M, Lagercrantz $\mathrm{H}$ (1979) Amniotic-fluid Acetylcholinesterase as a possible diagnostic test for neural-tube defects in early pregnancy. Lancet I:685-690

Sutherland GR, Brock DJH, Scrimgeour JB (1975) Amniotic fluid macrophages and the antenatal diagnosis of anencephaly and spina bifida. J Med Genet 12:135-137

Voigtländer T, Müller U, Schroeder TM (1978) Rapidly adhering cells in two cases of anencephaly. Hum Genet 45: $107-110$

Wald NJ, Cuckle HS (1979) Amniotic-fluid alpha-fetoprotein measurement in antenatal diagnosis of anencephaly and open spina bifida in early pregnancy. Second report of the U.K. collaborative study on alpha-fetoprotein in relation to neural-tube defects. Lancet II:651-662

Received November 10, 1980

\section{Note Added in Proof}

After this work had been finished we received notice on a similar work "Glial origin of rapidly adhering amniotic fluid cells" by P. Aula, H.v. Koskull, K. Teramo, O. Karjalainen, I. Virtanen, V. P. Lehto, and D. Dahl, Br Med J (in press) 